\title{
Hybrid algorithm for two-terminal reliability evaluation in communication networks
}

\author{
Musaria K. Mahmood ${ }^{1}$, Osman UCAN ${ }^{2}$, Zahraa Zaidan ${ }^{3}$, Sulaiman M. Karim ${ }^{4}$ \\ ${ }^{1}$ Istanbul Gelisim University, Turkey \\ ${ }^{2,3}$ Altinbas University, Turkey \\ ${ }^{4}$ Karabuk University, Turkey
}

\begin{tabular}{l} 
Article Info \\
\hline Article history: \\
Received Jul 12, 2020 \\
Revised Sep 7, 2020 \\
Accepted Sep 27, 2020 \\
\hline
\end{tabular}

\section{Keywords:}

Graph reduction

Hybrid algorithm

Tie-set

Two-terminal reliability

\begin{abstract}
Network reliability is valuable in establishing a survivable communication network. Reliability evaluation algorithms are used in the design stage and during the network deployment. This work presents a new multistage hybrid technique for two-terminal reliability evaluation problem. It is based on a combination of graph reduction techniques and tie-set method. A new approach has been introduced for deducing tie-sets in a network containing both unidirectional and bi-directional edges. The proposed algorithm can be applied for both simple and complex networks without restrictions. The results confirm that new algorithm evaluates network's reliability with decreasing computing time compared to classical algorithms. The results for a case study of a 20 -node network have demonstrated that the required time for reliability evaluation is decreased from ( $t>1$ hour) in the case of using a classical algorithm, to $(\mathrm{t}<1$ second) for the new algorithm.
\end{abstract}

This is an open access article under the CC BY-SA license.

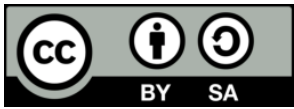

\section{Corresponding Author:}

Musaria Karim Mahmood

Department of Electrical and Electronics Engineering

Istanbul Gelisim University

Cihangir, Şehit Jandarma Komando Er, J. Kom. Er Hakan Öner Sk. No:1, 34310 Avcılar/İstanbul

Email:mkmahmood@gelisim.edu.tr

\section{INTRODUCTION}

Nowadays, data communication systems and especially computer networks are the major characteristic of the modern world technology. The network reliability is one of the important Quality of Service (QoS) factors beside security, availability, and delay. Network reliability can be defined as the probability of performing the mentioned functionality of a network in a successful way [1]. A further definition of the network reliability states that it is the probability of achieving successful communication operation between the transmitter and the receiver in a network [2]. Thus, high reliability has become an inevitable consequence for various controlled applications such as military, aircraft systems, and banking systems, where faults may cause financial and human life damages [3]. The network reliability requirement depends on the application and type of network such as wired network [4], wireless sensor network [5], mobile system [6], electrical distribution networks [7], and the electrical grid reliability assessment [8, 9]. The network reliability is classified into three types according to the number of involved source-destination nodes. The first, is the two-terminal reliability, concerning the measurement of the reliability between one source and one destination node. This problem has been treated widely because it is the basic of other reliability types [10-12]. The second form is the all-terminal reliability where all nodes are considered as possible source-destination pair as presented in [13]. Finally, the most general term is the k-terminal reliability where the problem is defined according to the value of $\mathrm{k}[14,15]$. Various techniques and 
algorithms are availables for computing the network reliability, including state space decomposition [16], Graph Reduction Technique (GRT) [17], cut-sets, tie-sets [18], Monte Carlo method [19], and conditional probability and connection matrix techniques [20]. Some of the mentioned methods may fail depending on the complexity of the network, the required accuracy, and the algorithm convergence speed. Different attempts have been continuously exerted to the development of new exact algorithms as in [21], or approximated methods algorithms as presented in [22, 23]. Exact methods are usually preferred when the purpose is an accurate calculation of static network's reliability, whereas approximation becomes the best solution in the case of dynamic network topology, as in military or mobile networks in general. The combination of more than one method can improve the reliability evaluation process by taking the advantages of each basic method used as components [24]. The major factors affecting the network availability from different layers are classified in order to indicate their impact. A review has recently been published concerning the probability methods in [25], where the most important trends and achievements in network reliability algorithms are presented.

The present work proposes a new algorithm for two-terminal reliability calculation; the Multistage Hybrid Technique (MHT), which is based on the successive application of two known techniques: the GRT, and the Tie-Set Method (TSM). The application of the algorithm is simulated by MATLAB with a complex network of 20-node and the results are compared with those obtained from the well-known classical tie-set algorithm. The new algorithm has proved its capability for real time reliability evaluation of random networks. The remaining parts of this paper are organized as follows. Section 2 introduces the basic theoretical backgrounds. Section 3 presents the research method and the MHT algorithm steps. The results from algorithm simulation are discussed in Section 4. Finally, Section 5 concludes this work.

\section{PRELIMINARIES}

\subsection{Network modeling}

The communication network $(\mathrm{CN})$ is one of the many physical problems that can be modeled graphically in order to be treated easily during design and enhancement phases. Any CN consists basically of computer devices, communication links, routers, switches, and other components connected together. It can be represented by an equivalent graph $\mathrm{G}=(\mathrm{N}, \mathrm{E})$, where $\mathrm{N}\left(n_{1}, n_{2}, \ldots n_{k}\right)$ is the set of k-nodes and $\mathrm{E}$ $\left(e_{1}, e_{2}, \ldots e_{m}\right)$ is a set of m-links. Each link $e_{i}$ has two states i.e. operational state with probability $p_{i}$, and failing state with probability $q_{i}=1-p_{i}$. The network topology can be presented as a matrix where element $L_{i k}$, represents the probability of a link between nodes $n_{i}$, and $n_{k}$. The diagonal elements give nodes probability. Assuming that the network satisfies the following assumptions:

a) Perfectly reliable nodes by the use of redundant materials $\left(L_{i i}=1\right)$,

b) Network components fail independently,

c) Each edge is either in working state or in failing state with known constant discrete probability.

The assumption of perfect nodes will not be negatively reflected to the generality of the proposed algorithm because a non-perfect node affects only the composition of connectivity matrix. A non-perfect node is replaced by two perfect nodes with a link between then with a probability equal to the probability of the original non-perfect node. This will increase the dimension of the connectivity matrix by one.

\subsection{TSM and GRT theoretical principles}

A tie-set is a group of network components with the property that if all components are in an operating state, then there is a path between the source node $n_{s}$ to the target node $n_{d}$. If none of its components can be removed without the loss of the above property, then the tie-set is minimal [26]. TSM consists of listing all minimal tie-set, and followed by the application of the inclusion equation called Poincare equation. $\left(T_{m}\right)$, is defined as the group of successive links forming the minimal path between $n_{s}$, and $n_{d}$. If there are $(i)$ tie-sets, then the two-terminal reliability is given by:

$$
R_{s d}=P\left(T_{1} \cup T_{2} \cup T_{3} \ldots \ldots \cup T_{i}\right)
$$

Where $P$ is the event probability If the tie sets are, all disjoints or mutually exclusive, then (1) can be written as

$$
R_{s d}=P\left(T_{1}\right)+P\left(T_{2}\right)+P\left(T_{3}\right)+\cdots+P\left(T_{i}\right)
$$

Since tie sets are not disjoint events in general, then (1) can be written as [27]:

$$
R_{s d}=P\left(T_{1}+T_{2}+T_{3}+\cdots+T_{i}\right)
$$




$$
\begin{aligned}
& =\left[P\left(T_{1}\right)+P\left(T_{2}\right)+P\left(T_{3}\right)+\cdots+P\left(T_{i}\right)\right]-\left[P\left(T_{1} T_{2}\right)+P\left(T_{1} T_{3}\right)+\ldots P\left(T_{a} T_{b}\right)_{a \neq b}\right]+ \\
& {\left[P\left(T_{1} T_{2} T_{3}\right)+P\left(T_{1} T_{3} T_{4}\right)+\cdots+P\left(T_{b} T_{a} T_{n}\right)_{b \neq a \neq n}+\ldots+(-1)^{\mathrm{i}-1}\left[P\left(T_{1} T_{2} T_{3} \ldots . T_{i}\right)\right]\right.}
\end{aligned}
$$

The difficulty of the TSM comes with tie sets enumeration algorithm and the application of Poincare equation, especially when the number and complexity of tie sets increase as a network become complex. To reduce the network complixity, GRT algorithm based on a simple parallel and series simplification is used as first step before the application of TSM. The CN connectivity usually shows an excessive parallel and series connection which promote the use of such simplification. Series and parallel simplifications are applied to the graph as as shown in Figure 1. The nodes $t, m$, and $c$ are connected in series where $m$ can be removed and be replaced by a direct link between $t$ and $c$ with the following probability:

$$
P_{s}=P_{1} \times P_{2}
$$
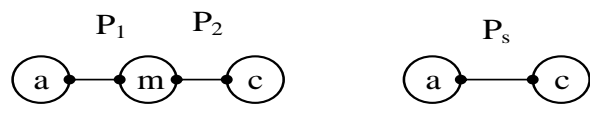

(a) Series

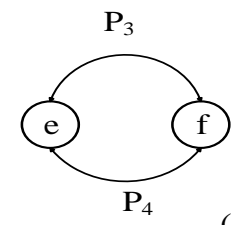

(b) Parallel

Figure 1. Series and parallel simplifications, (a) series, (b) parallel

Two parallel links between node (e) and node (f) are simplified and may be replaced by one link with probability $P_{p}$ :

$$
P_{p}=P_{3}+P_{4}=1-q_{3} \times q_{4}
$$

\section{REASEARCH METHOD}

MHT is accomplished by three stages, where each stage is composed of a number of sub-stages. The initialization is the first stage, followed by the application of GRT. Finally, all the minimal tie-sets are deduced, and the reliability is evaluated by the TSM.

\subsection{Initialization stage}

The $\mathrm{N}$-node $\mathrm{CN}$ topology can be presented with a three-dimensional connectivity matrix, $M(N, N, t)$. Depending on the maximum number of parallel edges between two communicated nodes, the value of $(t)$ is determined. For example, if there are maximum of four parallel links between two specific nodes, then $(t=4)$. However, if the network has no parallel links, $(t)$ is taken to be equal to 2 and this is to resolve the problem arising from the birth of new parallel links after the application of series reduction stage. In this case, the elements of $M_{N, N, 1}$ matrix represent the actual edges of the network, while all the elements of $M_{N, N, 2}$ matrix are set to zeros. Each element in $M$ represents one edge probability of operating successfully $\left(p_{i j k}\right)$. The elements of $M_{N, N, 2}$ describe "only" the parallel edge probabilities of being up and set to " 0 " for the rest of elements. Matrix $M$ can be considered as multi-layer matrix where each layer is $(N \times N)$ matrix with:

a) $0 \leq p_{i j k} \leq 1$,

b) If $i=j, p_{i i k}$ represents the diagonal elements which is the probability of node $n_{i}$ ( 1 for perferct nodes),

c) $1 \leq k \leq t$, and $t \geq 2$, and each matrix layer is defined by:

$$
M_{i, j, k}=\left[\begin{array}{ccc}
p_{11 k} & \cdots & p_{N 1 k} \\
\vdots & \ddots & \vdots \\
p_{N 1 k} & \cdots & p_{N N k}
\end{array}\right]
$$

\subsection{Parallel reduction procedure}

The first step is to test the third dimension of $M$ matrix in order to decide with which layer the reduction will start. Hence, the second layer has to be checked for any parallel edge. If $M_{i j 2}$ is a zero matrix, 
then there are no parallel links and series reduction can be directly applied. If at least one element of $M_{i j 2}$ matrix is not null, then a parallel reduction is required. For all elements of $\mathrm{M}$ matrix, if $p_{j i 2} \neq 0$, then:

$$
\begin{aligned}
& p_{i j 1}=p_{i j 1} \cup p_{i j 2}=p_{i j 1}+p_{i j 2}=1-\left(q_{i j 1} \times q_{i j 2}\right) \\
& p_{i j 2}=0
\end{aligned}
$$

In case $(t>2)$ parallel edges in the last layer matrix $M_{i, j, t>2}$ are treated firstly by considering layer $M_{i, j, t-1 \geq 2}$ as the base layer. After applying parallel reduction procedure on the two previous matrices, the first one is deleted (all its elements become zeros), while the other one is updated to contain the new calculated values. The flowchart of the parallel reduction procedure is illustrated in the Figure 2(a).

\subsection{Series reduction procedure}

The series reduction technique is applied after the parallel procedure as shown in Figure 2(b). It starts by checking the initial condition of node validation that is, the node $n_{i}$ is neither a source node $n_{s}$ nor a destination node $n_{d}$. The connectivity of $n_{i}$ is inspected from $M$. If $n_{i}$ is connected to only two terminal nodes $n_{j}$ and $n_{m}$, then it is removed from the graph by series simplification and the dimension of $M$ is decreased by eliminating row $(i)$, and column $(i)$. The application of the following equations declares the addition of a new link between $n_{j}$ and $n_{m}$ and the removal of node $n_{i}$.

$$
\begin{aligned}
& P_{j, m, 1}=P_{j, i, 1} \times P_{i, m, 1} \\
& P_{j, i, 1}=P_{i, m, 1}=0
\end{aligned}
$$

There is a possibility of new addition of parallel links between $n_{j}$, and $n_{m}$ to be checked where the parallel reduction must be repeated to resolve this problem before continuing. This is done via the inspection of the parallel_index number.

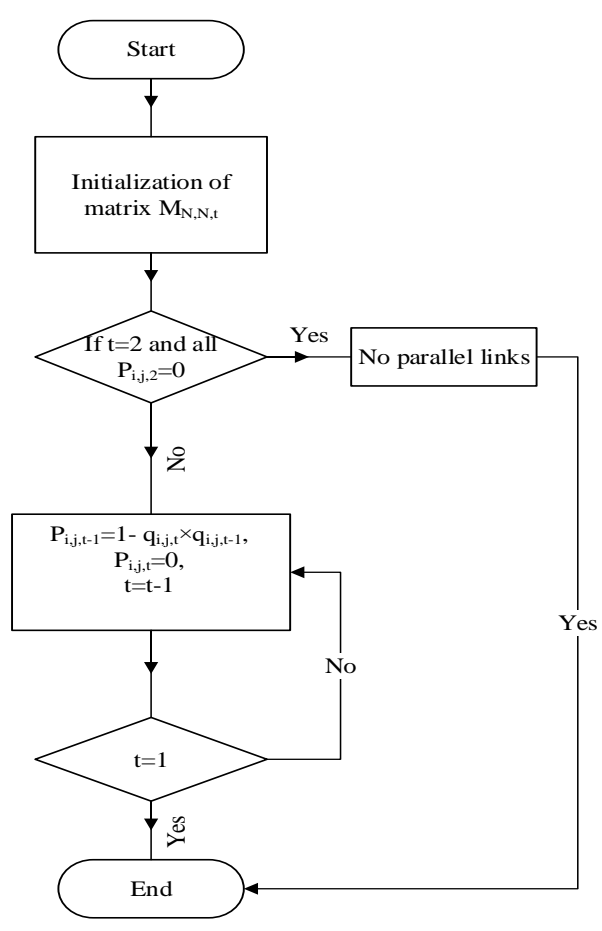

(a) Parallel reduction algorithm

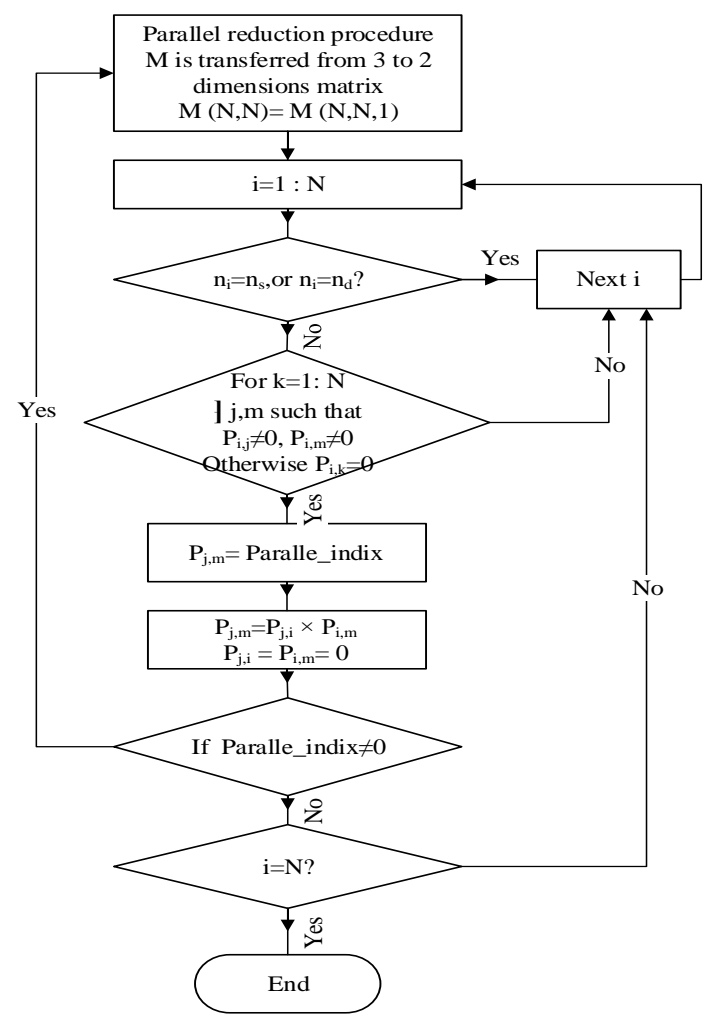

(b) Series reduction algorithm

Figure 2. Parallel and series reduction algorithm 


\subsection{Tie-sets generation}

On completing the reduction stage the matrix $M_{N, N, t}$ is converted into two-dimensional matrix $R_{N r, N r}$ which can be used to generate arrangement matrix, $T\left(v, N_{r}\right)$, where $\left(N_{r}\right)$ is the number of nodes after the application of GRT, and $v$ is the number of different arrangements for $\left(N_{r}-1\right)$ nodes (excluding $\left.n_{s}\right)$, that is:

$$
v=(N r-1) !
$$

The tie-sets generation step starts by denoting the source node $n_{s}$, and the destination node $n_{d}$, then seeking for all the possible minimum paths connecting the pair $\left(n_{s}, n_{d}\right)$. A single route may be composed of one or a group of edges and nodes. Since the remaining nodes $\mathrm{Nr}$ are less than the original number of nodes before reduction, the arrangement operation does not demand a very large memory or consuming a long processing time. Matrix $T$ is formed by enumerating all possible combinations of the remaining $(N r-1)$ nodes. The elements of $T$ are node numbers giving the location of links between nodes in the matrix $R$. The first column is filled with all elements that are equal to the number of the source node $n_{s}$. For example: if $T_{3,2}=5$, and $T_{3,3}=3$, it means that node number (5) is located in rows (3) and column number (2) in the $T$ matrix, and the element after (in the same row) is node number (3). In order to find the corresponding link probability between node (5), and node (3), element $R[5,3]=P_{5,3}$, is copied from matrix $R$. If $P_{i k}=0$, then there is no direct connection between node $n_{i}$, and $n_{k}$. An action is made by replacing node $n_{k}$ by $n_{i}$ in matrix $T$. This measure ensures the correctness of the connection check operation since no possible connecting case will be skipped. In order to get the correct sequence for each tie-set, the following three simplification steps have to be followed:

a) Eliminate all the repeated nodes in one row,

b) Remove each node after the destination $n_{d}$, and

c) Eliminate redundant tie-sets.

Finally, we will get a matrix $T S\left(t_{t s}, t_{r}\right)$, which contains the entire tie-sets. The number of rows $t_{t s}$, is equal to the number of the tie-sets, while the number $t_{r}$ is the node number in a specific tie-set with $\left(t_{r} \leq\right.$ $\mathrm{Nr}$ ). Figure 3 depicts tie-set generation procedure.

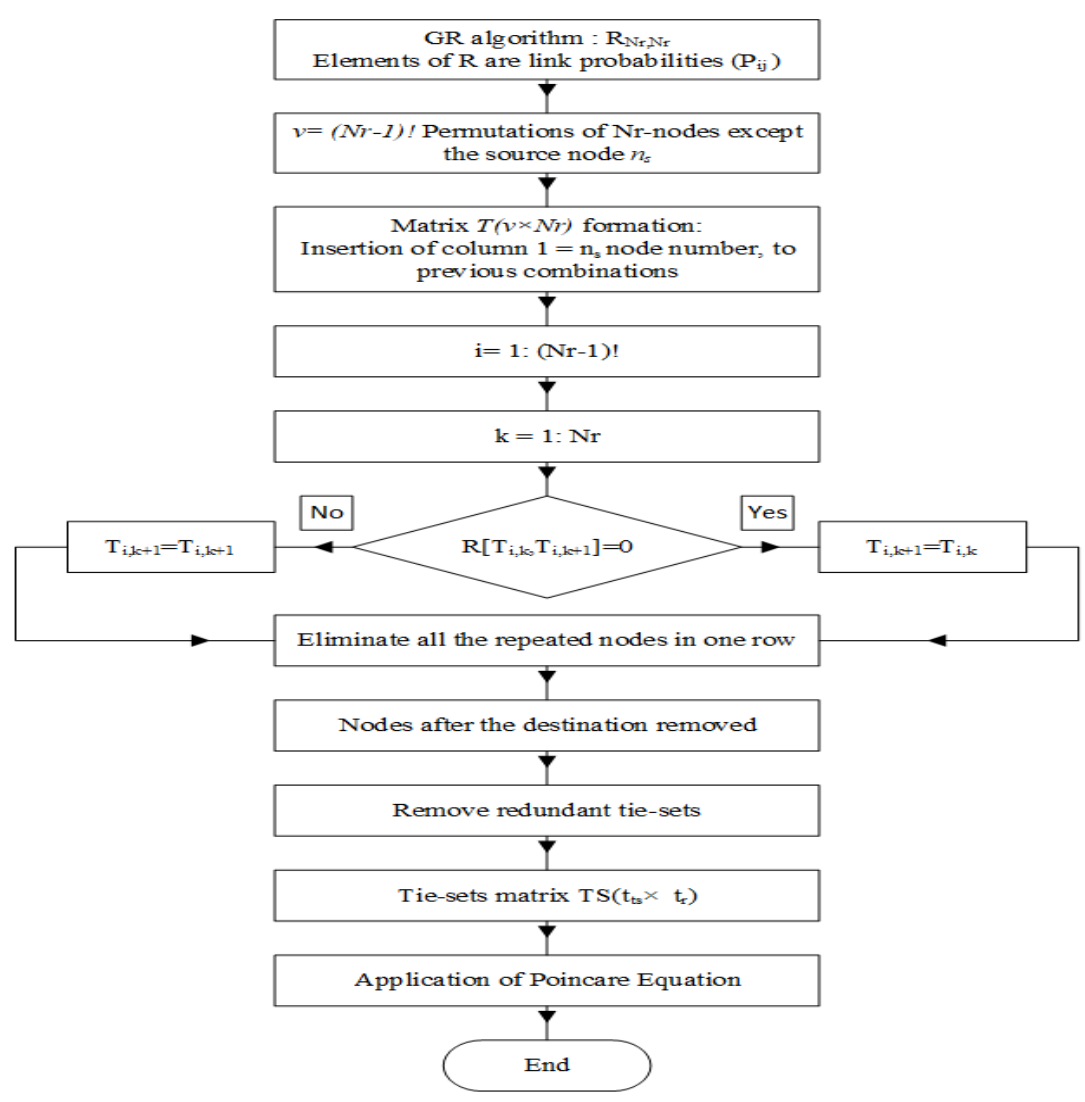

Figure 3. Tie-sets generation algorithm 


\section{RESULT AND DESCUSSION}

The MHT performance is evaluated by a 20-node random topology implementation as shown in Figure 4. The topology is simulated using $P_{i k}=0.9$ as link probability, and perfect nodes. For clarity, only a sample of the results is listed in Table 1 , where nodes $\left(n_{1}, n_{2}, n_{3}, n_{4}\right)$ are considered successively as source nodes for all possible destinations. The results of the MHT Algorithm are compared against existing classical tie-sets algorithms. $\mathrm{S}$ is the source node, while $\mathrm{D}$ is the destination node. $\mathrm{R}$ is the reliability, which must be the same for both simulated algorithms. $M_{p H}$, and $T_{H}(s)$ are respectively the number of tie-sets and time required for reliability evaluation for the new MHT algorithm. $M_{p i}$, and $T_{i}(s)$ are the same variables for the classical tie-sets algorithms, the Path Tracing Algorithm (PTA). The results show a clear improvement by a clear reduction in the number of tie-sets using MHT compared to PTA. This decrease will have a direct impact on decreasing the computing time required for reliability evaluation. For example, for the commodity $\left(\mathrm{S}=n_{1} ; \mathrm{D}=n_{2}\right)$, the number of tie-set has decreased from (30) to (8) as shown in the first row of Table 1. This decrease is positively reflected in the computing time required for reliability evaluation. This time is reduced from $(t>1$ Hour $)$ in PTA to $(0.042 \mathrm{sec})$ when MHT is applied. For complicated networks with high node number, the improvement is expected to be much more significant.

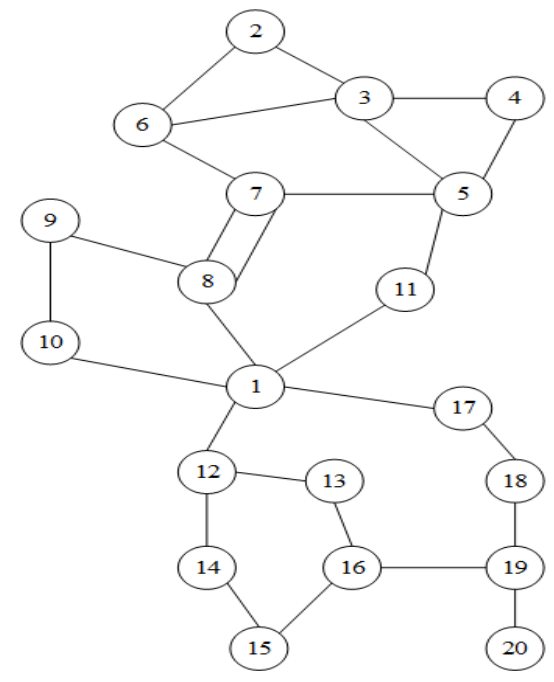

Figure 4. Simulated topology

Table 1. Sumilation results

\begin{tabular}{cccccccccccccc}
\hline $\mathrm{S}$ & $\mathrm{D}$ & $\boldsymbol{M} \boldsymbol{p}_{\boldsymbol{H}}$ & $\boldsymbol{T}_{\boldsymbol{H}}(\mathrm{s})$ & $\boldsymbol{M} \boldsymbol{p}_{\boldsymbol{t}}$ & $\boldsymbol{T}_{\boldsymbol{t}}(\mathrm{s})$ & $\mathrm{R}$ & $\mathrm{S}$ & $\mathrm{D}$ & $\boldsymbol{M} \boldsymbol{p}_{\boldsymbol{H}}$ & $\boldsymbol{T}_{\boldsymbol{H}}(\mathrm{s})$ & $\boldsymbol{M} \boldsymbol{p}_{\boldsymbol{t}}$ & $\boldsymbol{T}_{\boldsymbol{t}}(\mathrm{s})$ & $\mathrm{R}$ \\
\hline 1 & 2 & 8 & 0.042 & 30 & long & 0.9782 & 2 & 18 & 1 & 0.0451 & 1 & long & 0.9209 \\
1 & 3 & 4 & 0.0141 & 20 & long & 0.9886 & 2 & 19 & 1 & 0.0450 & 1 & long & 0.9168 \\
1 & 4 & 7 & 0.0408 & 28 & long & 0.9798 & 2 & 20 & 1 & 0.0442 & 1 & Long & 0.8251 \\
1 & 5 & 1 & 0.0176 & 21 & Long & 0.9906 & 3 & 4 & 1 & 0.0061 & 12 & 13.437 & 0.9888 \\
1 & 6 & 4 & 0.0079 & 25 & long & 0.9874 & 3 & 5 & 1 & 0.0076 & 12 & 14.227 & 0.9974 \\
1 & 7 & 1 & 0.0028 & 9 & 10.596 & 0.9926 & 3 & 6 & 1 & 0.0043 & 12 & 14.364 & 0.9974 \\
1 & 8 & 1 & 0.0027 & 12 & 13.5710 & 0.9943 & 3 & 7 & 1 & 0.0034 & 12 & 13.241 & 0.9953 \\
1 & 9 & 1 & 0.0029 & 12 & 12.7389 & 0.9774 & 3 & 8 & 4 & 0.0053 & 16 & 53.795 & 0.9932 \\
1 & 10 & 1 & 0.0019 & 12 & 12.5267 & 0.9793 & 3 & 9 & 8 & 0.0687 & 24 & Long & 0.9730 \\
1 & 11 & 1 & 0.0017 & 21 & long & 0.9855 & 3 & 10 & 8 & 0.0689 & 24 & Long & 0.9715 \\
1 & 12 & 2 & 0.0099 & 3 & 1.21065 & 0.9622 & 3 & 11 & 4 & 0.0055 & 20 & long & 0.9833 \\
1 & 13 & 4 & 0.0145 & 4 & 1.06430 & 0.9468 & 3 & 12 & 1 & 0.0239 & 1 & long & 0.9513 \\
1 & 14 & 4 & 0.0129 & 4 & 1.01374 & 0.9404 & 3 & 13 & 1 & 0.0291 & 1 & long & 0.9361 \\
1 & 15 & 4 & 0.0142 & 4 & 0.95241 & 0.9362 & 3 & 14 & 1 & 0.0277 & 1 & long & 0.9297 \\
1 & 16 & 2 & 0.0027 & 3 & 0.95085 & 0.9497 & 3 & 15 & 1 & 0.0275 & 1 & Long & 0.9256 \\
1 & 17 & 2 & 0.0027 & 3 & 1.04519 & 0.9622 & 3 & 16 & 1 & 0.0169 & 1 & long & 0.9389 \\
1 & 18 & 2 & 0.0026 & 3 & 0.97193 & 0.9414 & 3 & 17 & 1 & 0.0170 & 1 & long & 0.9513 \\
1 & 19 & 1 & 0.0024 & 3 & 0.94365 & 0.9372 & 3 & 18 & 1 & 0.0168 & 1 & long & 0.9307 \\
1 & 20 & 1 & 0.0023 & 3 & 0.94788 & 0.8435 & 3 & 19 & 1 & 0.0167 & 1 & long & 0.9266 \\
2 & 3 & 1 & 0.3155 & 12 & 13.5680 & 0.9888 & 3 & 20 & 1 & 0.0159 & 1 & Long & 0.8339 \\
2 & 4 & 7 & 0.4314 & 19 & 894.000 & 0.9778 & 4 & 6 & 4 & 0.0213 & 14 & 17.192 & 0.9865 \\
2 & 5 & 4 & 0.0293 & 15 & 19.2411 & 0.9865 & 4 & 7 & 4 & 0.0155 & 14 & 16.325 & 0.9863 \\
2 & 6 & 1 & 0.0136 & 11 & 14.8627 & 0.9888 & 4 & 8 & 7 & 0.0419 & 20 & long & 0.9843 \\
2 & 7 & 4 & 0.0234 & 22 & $10 n g$ & 0.9848 & 4 & 9 & 14 & 7.1935 & 32 & long & 0.9642
\end{tabular}




\begin{tabular}{cccccccccccccc}
\hline $\mathrm{S}$ & $\mathrm{D}$ & $\boldsymbol{M} \boldsymbol{p}_{\boldsymbol{H}}$ & $\boldsymbol{T}_{\boldsymbol{H}}(\mathrm{s})$ & $\boldsymbol{M} \boldsymbol{p}_{\boldsymbol{t}}$ & $\boldsymbol{T}_{\boldsymbol{t}}(\mathrm{s})$ & $\mathrm{R}$ & $\mathrm{S}$ & $\mathrm{D}$ & $\boldsymbol{M} \boldsymbol{p}_{\boldsymbol{H}}$ & $\boldsymbol{T}_{\boldsymbol{H}}(\mathrm{s})$ & $\boldsymbol{M} \boldsymbol{p}_{\boldsymbol{t}}$ & $\boldsymbol{T}_{\boldsymbol{t}}(\mathrm{s})$ & $\mathrm{R}$ \\
\hline 2 & 8 & 8 & 0.1385 & 24 & long & 0.9828 & 4 & 10 & 14 & 7.1787 & 32 & long & 0.9627 \\
2 & 9 & 16 & 39.8852 & 36 & long & 0.9628 & 4 & 11 & 7 & 0.0393 & 28 & long & 0.9746 \\
2 & 10 & 16 & 40.7743 & 36 & long & 0.9612 & 4 & 13 & 1 & 0.0546 & 1 & long & 0.92764 \\
2 & 11 & 8 & 0.1352 & 30 & long & 0.9727 & 4 & 14 & 1 & 0.0532 & 1 & long & 0.92133 \\
2 & 12 & 1 & 0.0521 & 1 & long & 0.9413 & 4 & 15 & 1 & 0.0530 & 1 & Long & 0.91724 \\
2 & 13 & 1 & 0.0574 & 1 & long & 0.9262 & 4 & 16 & 1 & 0.0424 & 1 & long & 0.93045 \\
2 & 14 & 1 & 0.0560 & 1 & long & 0.9199 & 4 & 17 & 1 & 0.0425 & 1 & long & 0.94275 \\
2 & 15 & 1 & 0.0558 & 1 & Long & 0.9158 & 4 & 18 & 1 & 0.0423 & 1 & long & 0.92232 \\
2 & 16 & 1 & 0.0452 & 1 & long & 0.9290 & 4 & 19 & 1 & 0.0422 & 1 & long & 0.91823 \\
2 & 17 & 1 & 0.0452 & 1 & long & 0.9413 & 4 & 20 & 1 & 0.0414 & 1 & Long & 0.82641 \\
\hline
\end{tabular}

Long: is more than one hour

\section{CONCLUSION}

Several methods have been proposed to compute network reliability evaluation. TSM, and GRT are very often used, especially in probabilistic context. Tie-set can treat small to medium complexity networks. GRT is used to evaluate all network types. In this paper, an efficient algorithm named MHT is proposed. The new algorithm has its efficiency due to the fact that it combines the advantages of both TSM and GRT algorithms, and can be applied to any network regardless of whether the network topology is simple, medium or complex. MHT efficiency is demonstrated by the application of GRT first, which yields to simplifying the network topology resulting a simple application of the TSM. The obtained simulation esults show a significant improvement in the required time for network reliability evaluation. In this context, MHT can be considered as a real-time tool for network reliability calculation.

\section{REFERENCES}

[1] M.K. Mahmood., "Development of new algorithm for communication networks reliability based on tie set method combined with a modified flooding algorithm," Tikrit Journal of Engineering Sciences, vol. 20, no. 1, pp. 10-20, 2013.

[2] P. Zhu, J. Han, Y. Guo, and F. Lombardi, "Reliability and criticality analysis of communication networks by stochastic computation," IEEE Network, vol. 30, no. 6, pp. 70-76, 2016.

[3] A. P. Guimaraes, H. M. N. Oliveira, R. Barros. and, P. R. M Maciel, "Availability analysis of redundant computer networks: A strategy based on reliability importance," IEEE $3^{\text {rd }}$ International Conference on Communication Software and Networks, China, pp. 328-332, 2011.

[4] A. M. Shooman., "Methods for communication-network reliability analysis: probabilistic graph reduction," Annual Reliability and Maintainability Symposium, pp. 441-448, 1992.

[5] Q. Liu., "Coverage reliability evaluation of wireless sensor network considering common cause failures based on d-s evidence theor," IEEE Transactions on reliability, pp. 1-15, 2020.

[6] V. K. Singh, P. Samundiswary and M. Sivasindhu, "Cluster based reliable communication for 5G network," International Conference on Communication and Signal Processing, India, pp. 853-856, 2019.

[7] K. B. Kela, B. N. Suthar, and L. D. Arya, "Reliability optimization of electrical distribution systems considering expenditures on maintenance and customer interruptions," Indonesian Journal of Electrical Engineering and Computer Science, vol. 14, no.3, pp. 1057-1064, 2019.

[8] A. M. Agwa, et al., "Electrical grid reliability assessment by fault tree analysis," Indonesian Journal of Electrical Engineering and Computer Science, vol. 17. no. 3, pp. 1127-1134, 2020.

[9] M. A. Al-shehhri, Y. Guo, and G. Lei, "A systematic review of reliability studies of grid-connected renewable energy microgrids," $2^{\text {nd }}$ International Conference on Electrical, Communication and Computer Engineering, Istanbul, Turkey, pp. 1-6, 2020.

[10] M. Le, M. Walter and J. Weidendorfer, "Improving the Kuo-Lu-Yeh algorithm for assessing two-terminal reliability," European Dependable Computing Conference, Newcastle, UK, pp. 13-22, 2014.

[11] F. Majid, and H.B. Luiz," Finding all the lower boundary points in a multistate two-terminal network," IEEE Transactions on Reliability, vol. 66, pp. 677-688, 2017.

[12] M. K. Mahmood, F. M. A. Naima, and L. S. Abdulla, "An efficient multi-stages algorithm for the determination of communication network reliability," International Journal of Computers and Communications, vol. 9, pp. 36-43, 2015.

[13] J. Silva, T. Gomes, D. Tipper, L. Martins, and V. Kounev, "An algorithm for computing all-terminal reliability bounds," $6^{\text {th }}$ International Workshop on Reliable Networks Design and Modeling, Barcelona, Spain, pp. 76-83, 2014.

[14] Eryilmaz, S. and Bozbulut, A. R., "An algorithmic approach for the dynamic reliability analysis of non-repairable multi-state weighted k-out-of-n: G system," Reliability Engineering and System Safety, vol. 131, pp. 61-65, 2014.

[15] X. Shihu and Y. Jun, "k-Terminal Reliability of ad hoc networks considering the impacts of node failures and interference," IEEE Transactions on Reliability, vol. 69 no. 2, pp. 725-739, 2020.

[16] G. Bai, et al.," An improved method for reliability evaluation of two-terminal multistate networks based on state space decomposition," IEEE Transactions on reliability, pp. 1-12, 2020. 
[17] P. L'Ecuyer, S. Saggadi, and B. Tuffin, "Graph reductions to speed up importance sampling-based static reliability estimation," 2011 Winter Simulation Conference, Phoenix, AZ, USA, pp. 429-438, 2011.

[18] M. K. Mahmood, and I. Myderrizi, "Reliability evaluation using a clustering technique based on tie-set method,"

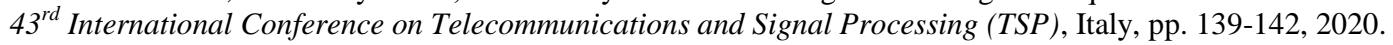

[19] P. Praks, et al.," Monte-Carlo based reliability modelling of a gas network using graph theory approach," $9^{\text {th }}$ International Conference on Availability, Reliability and Security, Fribourg, Switzerland, pp. 380-386, 2014.

[20] M. F. Firuzabad, R. Billinton, T.S. Munian, and B. Vinayagam, "A novel approach to determine minimal tie-sets of complex networks," IEEE Transactions on Reliability, vol. 53, no. 1, pp. 61-70, 2004.

[21] R. Dong, Y. Zhu, Z. Xu, and F. Li, "Decision diagram based symbolic algorithm for evaluating the reliability of a multistate flow network mathematical problems in engineering," Mathematical Problems in Engineering, pp. 1-13, 2016.

[22] A. Rai, R. C. Valenzuela, B. Tuffin, and P. Dersin, "Approximate Zero-Variance importance sampling for static network reliability estimation with node failures and application to rail systems," Winter Simulation Conference, Washington, DC, USA, pp. 3201-3212, 2016.

[23] C. Bhargava, and R. Loka, "An open source tool for reliability evaluation of distribution system using Monte Carlo simulation," Indonesian Journal of Electrical Engineering and Computer Science, vol. 14. no. 3, pp. 1065-1075, 2019.

[24] D. Zhang, et al., "Hybrid learning algorithm of radial basis function networks for reliability analysis," IEEE Transactions on reliability, pp. 1-14, 2020.

[25] V. Gaur, et al., "A review of metrics, algorithms and methodologies for network reliability," IEEE International Conference on Industrial Engineering and Engineering Management, Macao, pp. 1129-1133, 2019.

[26] Malinowski, J.," A new efficient algorithm for generating all minimal tie-sets connecting selected nodes in a mesh structured network," IEEE Transactions on Reliability, vol. 59. no.1, pp. 203-211, 2010.

[27] Musaria K. Mahmood, Fawzi M. Al-Naima, and Zahraa Zaidan, "Reliability assessment of the Iraqi National communication network," Indonesian Journal of Electrical Engineering and Informatics, vol. 6, no. 4. pp. 448-457, 2018. 